\title{
Research on Maneuvering Flight Trail Simulation of Air Combat
}

\author{
Qi-ke $\mathrm{Wu}^{1, \mathrm{a}}$, Deng-Kai Yao ${ }^{1, \mathrm{~b}}$, Gu-hao Zhao ${ }^{1, \mathrm{~b}}$, Tian-chi Sun ${ }^{1, \mathrm{~b}}$
}

${ }^{1}$ National Key Laboratory of Air Traffic Collision Prevention, Air Traffic Control and Navigation College, Air Force Engineering University, Xi'an, Shaanxi, 710051, China

àwuqiklove@163.com, ${ }^{b} 308541465 @ q q . c o m$

\section{Keywords: Spiral; Flight Trail; Coordinates Transformation; Simulation Model}

\begin{abstract}
Flight trail planning is a very important part of the airspace planning. The traditional flight trail planning was mostly based on kinematic equation and spatial geometric relationships while neglecting the wind disturbances. Refer to improve the accuracy and security of the airspace planning and reflect the actual fighter's trajectory with wind disturbances, this paper put forward an improvement on traditional flight trail planning by utilizing spiral. Considering detecting of radar and deviation of trail, the dynamic and kinematic models of fighter were established, and constructed the simulation model of maneuvering flight in three-dimensional space to emulate the fighter's direct real flight trail. The derivation and model indicate that the model proposed can directly simulate various flight trails in air combat, and provide the airspace planning with safe and reliable reference which is conditionally universal.
\end{abstract}

\section{Introduction}

Meeting the needs of transformation and construction of the air force combat power generation model, and relieving the outstanding problems of contradictions of China's airspace resource requirements effectively, has become a problem to be solved by military and civil aviation at present.

At present, scholars at home and abroad simplify the fighters at $2 \mathrm{~d}$ or $3 \mathrm{~d}$ motor to a uniform, uniform speed and variable speed movement ${ }^{[1-3]}$ combined with straight and curve lines and then, make a discussion of the solutions of motion equation ${ }^{[4]}$. Some scholars make the test of the flying ability $^{[5]}$ of air track according to some characteristics of the fighter to determine the trajectory. Also, some scholars put forward using SPL three times to fit trajectories of combat force and calculate the curvature of the orbit ${ }^{[6]}$. The orbit from the simulation of these methods accords with certain tactical background, the requirements of tactical intention, but it ignores the influence caused by factors like the unawareness motor of the pilots and the disturbance of the air ${ }^{[7]}$.It cannot reflect the actual movement characteristics disturbed by factors like air.

In the view of the safety of the delimited airspace under the background of the modern air combat, this article calculates the maximum distance of offset when turning based on the wind spiral method, so as to determine the shortest distance of training airspace transversely. The simulation results show that the track simulated by this method accords with the actual flying characteristics of the target.

\section{The principle of the track of the fighters at air combat}

\section{The model of the fighters dynamic}

In the air combat simulation, the flight simulation of the fighters often uses particle dynamic equations, so assuming that fighters don't sideslip and reasoning vectors are along the direction of velocity, so fighters' particle dynamics equations in the track coordinate system are as follows: 


$$
\left\{\begin{array}{l}
\frac{d v}{d t}=g\left(n_{x}-\sin \theta\right) \\
\frac{d \theta}{d t}=g\left(n_{f} \cos \gamma-\cos \theta\right) / v \\
\frac{d \phi}{d t}=-g n_{f} \sin \gamma /(v \cos \theta)
\end{array}\right.
$$

In the equation, $\mathrm{v}$ refers to the speed of the fighter; g refers to the acceleration of gravity; $n_{x}$ refers to the tangential overload; $n_{f}$ refers to the normal overload; $\theta$ refers to the pitching angle; $\phi$ refers to the course angle; $\gamma$ refers to the gradient.

The equations above all omit the sideslip angle $\beta$ and the corresponding lateral force. The equations above can get the aircraft's $v 、 \theta$ and $\phi$ at $t_{i}$ by using integral.

\section{The model of the fighters kinematics}

The kinematics equations of the aircraft in the ground coordinate system are as follows:

$$
\left\{\begin{array}{l}
\frac{d x}{d t}=v \cos \theta \cos \phi \\
\frac{d y}{d t}=v \sin \theta \\
\frac{d v}{d t}=-v \cos \theta \sin \phi
\end{array}\right.
$$

Integrating the aircraft dynamic equation and use the location at the time of $t_{i}$ we can get the equations of location at the time of $t_{i+1}$ :

$$
\left\{\begin{array}{l}
X_{i+1}=X_{i}+V_{i} \cos \theta_{i} \cos \phi_{i} \Delta t \\
Y_{i+1}=Y_{i}+V_{i} \sin \theta_{i} \Delta t \\
Z_{i+1}=Z_{i}-V_{i} \cos \theta_{i} \cos \phi_{i} \Delta t
\end{array}\right.
$$

Among these equations, $\Delta t=t_{i+1}-t_{i}$ refers to the time step in the simulation.

\section{The conditions of the radar finding targets related to the fighter's movement}

To establish mathematical systems from the aspects of the radar's detection range the location of the target related to the radar, posture and so on.

Condition 1 the altitude and speed for radar to find the target should be

$$
\left\{\begin{array}{l}
H_{t \min } \leq H_{t} \leq H_{t \max } \\
V_{t_{\text {min }}} \leq V_{t} \leq V_{t \max } \\
\Delta H_{t_{\text {min }}} \leq \Delta H_{t} \leq \Delta H_{t \text { max }}
\end{array}\right.
$$

In the formula, $H_{t} 、 V_{t} 、 \Delta H_{t}$ are the altitude of the target, the speed of the target and the altitude intercept between the target and the fighter respectively; $H_{t \min } 、 H_{t \max }$ are the minimum and maximum flight level for radar to find the target; $V_{t \min } 、 V_{t \max }$ are the minimum and maximum speed for radar to find the target; $\Delta H_{t \min } 、 \Delta H_{t \max }$ are the minimum and maximum altitude intercept for radar to find the target.

Condition 2 the azimuth angle $\delta$ and the angle of pitch $\lambda$ of the target under the radar coordinate system should be

$\left\{\begin{array}{l}\delta_{\text {left }} \leq \delta \leq \delta_{\text {right }} \\ \lambda_{\text {bottom }} \leq \lambda \leq \lambda_{\text {top }}\end{array}\right.$

$\delta_{\text {left }} 、 \delta_{\text {right }} 、 \lambda_{\text {bottom }} 、 \lambda_{\text {top }}$ are the border of the azimuth angle and the pitch angle under the condition of instantaneous potential field of the radar respectively.

Condition 3 the angle rate to achieve the maximum turning $\omega$ should be:

$\omega \leq \omega_{\max }$

In this equation, $\omega_{\max }$ is the maximum angular velocity to achieve the goal of turning of the 
target.

Condition 4 the distance to the target should be

$R_{c} \leq D \leq R$ In the process of searching

$D \leq R_{c} \quad$ Under the condition of interception and tracing

In the equation, $R$ is the maximum distance for radar to find the target and $R_{c}$ is the maximum distance for the radar to intercept the target. The calculations of them are as follows:

$$
p_{d i}=1 /\left[1+\left(c_{2} D^{4} / \sigma_{i}\right)\right]^{c_{1}}
$$

In the equation, $D$ is the vertical distance between the two aircrafts, $c_{1} 、 c_{2}$ are the parameters of the radar model; $\sigma$ 为 is the radar cross section area(RCS).

\section{Institutions Optimization Design}

The delimitation of the training airspace based on the wind spiral method

The mathematical essence of the wind spiral method can be regarded as Archimedes spiral, Shown as Fig1:

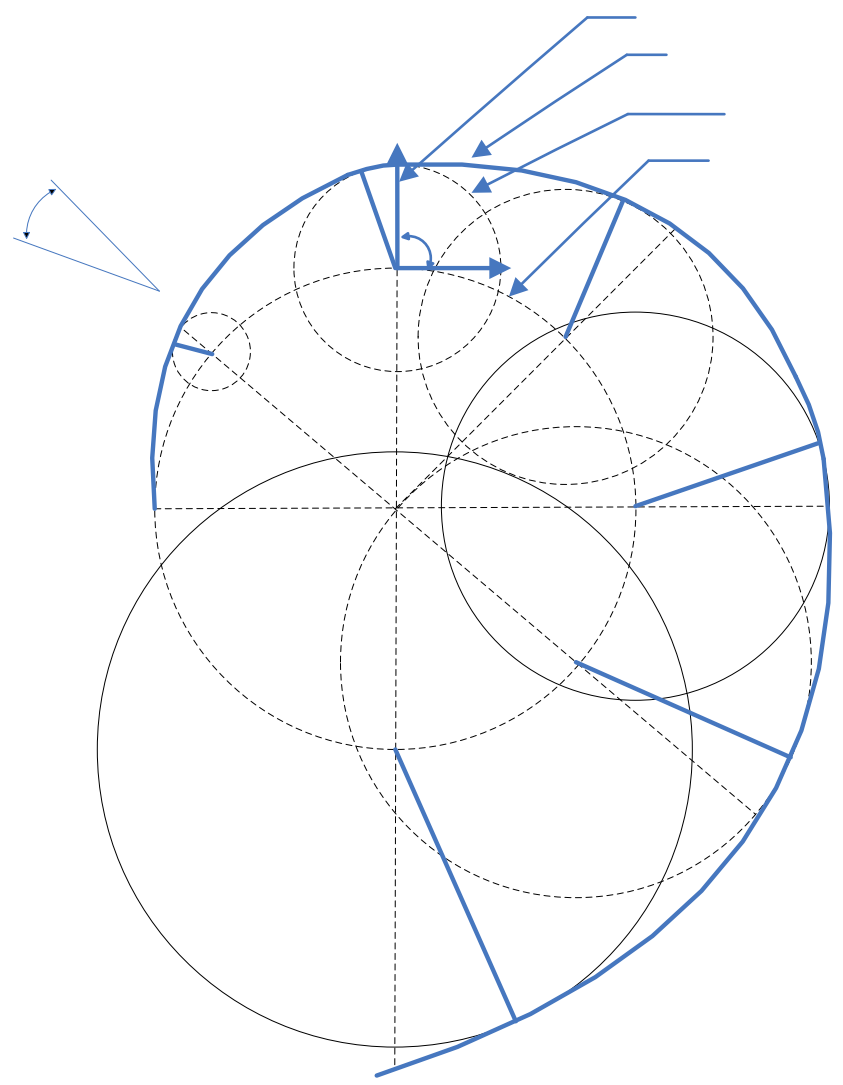

Fig.1.sketch map of the wind spiral

The detailed process of the wind spiral method applied to one-to-one air combat is shown as Fig1:

Step1: Assuming the maximum distance for the radar to detect is $R_{c}=40 \mathrm{~km}$ (after the head-on around tactics, the distance cannot be less than $40 \mathrm{~km}$ ), the shortest time of the second detection is $30 \mathrm{~s}$, and then making $40 \mathrm{~km}$ as the vertical distance of the finishing point of the maneuvering, and then determining the turning point, finally making sure the shortest vertical distance $\mathrm{L}$.

Step2: Flight level $6000 \mathrm{~m}, I A S=900 \mathrm{~km} / \mathrm{h}$, transforming into TAS and $\gamma=79^{\circ}$ under the standard condition, and making sure the required angle of maneuvering so as to select the unit angle.

Step3: Making sure the circumference of the ideal track according to the certain turning point and the turning radius.

Step4: Using $45^{\circ}$ as the unit interval, dividing the circumference into the equal parts and making 
sure the position of every data point $a, b 、 c 、 \ldots, n$.

Fig.2. the stimulated process of wind spirals

Step5: Making sure the speed of wind referring to the standard wind set in the ICAO8168 document and the linear relationship between the wind speed and the height with the increase of height in $0 \sim 7000 \mathrm{~m}$, the wind spirals are from the turning radius $\mathrm{r}$, getting from the increasing of the influence of the wind $E_{\rho}$ on the ideal track. The influence of the wind can be calculated in the equation:

$$
E_{\rho}=(\rho / \omega) \times(W / 3600)
$$

$\rho$ is the turning angle, $\omega$ is the turning angular rate of the aircraft, $W$ is the speed of the wind.

Specific construct steps are as follows:

In the place that is vertical to the windless radius $r$, by increasing the distance of $E_{\rho}$, locating b1 $\mathrm{c} 1 、 \ldots, \mathrm{n} 1$. Selecting point $\mathrm{O}$ as the center of the basic point and drawing the circuit with $\mathrm{r}$ as the radius. Setting $270^{\circ}$ as the standard and $45^{\circ}$ as the unit interval, at the $315^{\circ} 、 0^{\circ} 、 45^{\circ}$ 、 $90^{\circ} 、 135^{\circ} 、 180^{\circ}$ of the circuit, determining the a、b、c、d、e、f、n. Using each angle as the standard to calculate $E_{\rho}$, by increasing the distance of $E_{\rho}$, locating b1 $\mathrm{c} 1 、 \ldots, \mathrm{n} 1$.

Step6: calculating b2、c2、.., $n 2$, the $W / v$ before they locate in $b 1 、 c 1 、 \ldots, n 1$ and the distance between $\mathrm{b} 、 \mathrm{c} 、 \ldots, \mathrm{n}$ and them $E_{\rho}$.

The direction of $\mathrm{b} 2$ is the angle of $\mathrm{b} 1 \operatorname{minus} \arcsin (w / v)$ alongside the counterclockwise direction. Using the distance of $E_{\theta}$ whose length is b to decide the position of b2. The rest can be done in the same manner, so that we can get the position of 2 、... n2 .

The formed wind spiral starts from the point“a”, and locates at the windless radius throughb2、c2、... n2. The process of construct is showed as Graph 4. The shortest the unit intervals are, the more smooth the wind spirals are.

Step7: Connecting the wind spiral with the end of the track front and back to form a complete track. On the basis of the cruise track before maneuvering, calculating the longest vertical distance M skewing from the track.

This paper uses Matlab to stimulate, under the condition of regardless of the artificial operation, the type of aircrafts are the same between ours and the enemies'. Taking the example of one type of the foreign aircraft, setting the speed as $I A S=900 \mathrm{~km} / \mathrm{h}$, the pitch, turning radius, the turning time and corresponding parameters are shown in Table1:

Table.1. corresponding parameters of stimulation

\begin{tabular}{llllll}
\hline$I A S(\mathrm{~km} / \mathrm{h})$ & $n_{\mathrm{f}}(\mathrm{g})$ & $r(\mathrm{~m})$ & $\gamma\left({ }^{\circ}\right)$ & $\omega(\% / \mathrm{s})$ & $t(\mathrm{~s})$ \\
\hline 900 & 5.22 & 1371 & 79 & 11 & 32.8 \\
\hline
\end{tabular}

In the process of calculating, IAS should be transformed into true speed, and the true speed can 
be calculated according to the altitude and IAS. In the process of air combat, regardless of the change of the height of the aircraft, the paper assumes the height of the stimulation is $6000 \mathrm{~m}$.

In the turning, after finishing drawing the wind spirals, shown as Fig3, we need to draw its tangent line and the coordination of the track front and back. Because the parameters of the spirals radius are changeable, so when we decide the tangent point, we should calculate under the condition of the radius, rather than use the angle as the initial condition.

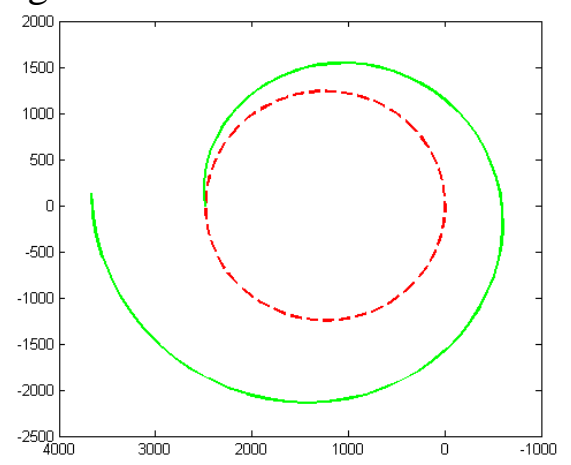

Fig.3. the stimulation of the wind spirals

When confronting with enemies, the aim of our aircrafts' maneuvering is often, both required to avoid the detective of other part's radars and tracing and attacking the enemies. For this, document 12 puts forward the head-on around tactics used by single aircraft air combat and the synergy around tactics used by coordinated air combat. The advantages of the tactics: getting the opportunity to attack in shorter time, taking full advantage of all-direction attacking missile, at the same time, avoiding attacked by enemies.

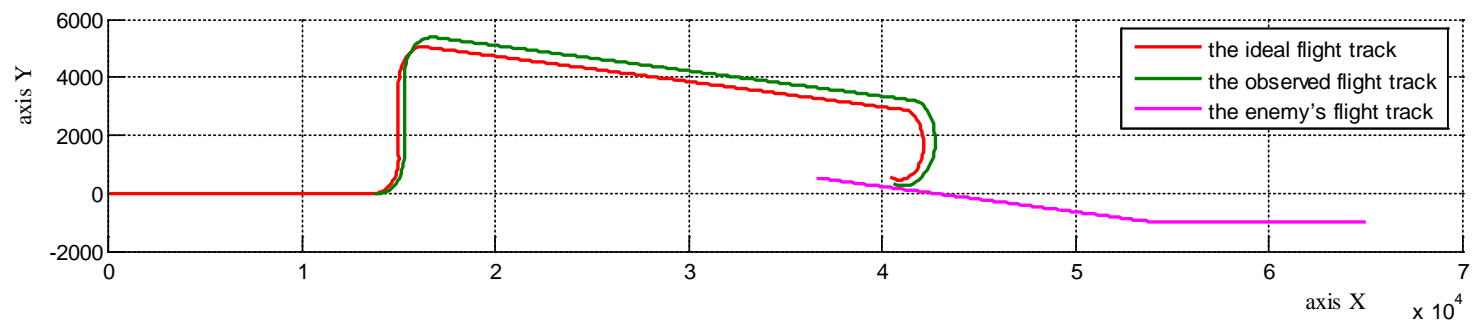

Fig.4. the vertical view of the motion trail of the air combat

(1) Fig4 shows us the comparison between the airspace areas before or after the improvement in the process of middle distance to short distance of the one-to-one air combat. From the comparison, we can see that the improved track alongside the $\mathrm{Y}$ direction completely covers the ideal track. Along the $\mathrm{X}$ direction, we use $40 \mathrm{~km}$ as the end of the first turning point of our part's aircraft to calculate the turning point, which shows us that the position is smaller than the position of the ideal track. That means that the airspace using the improved method can completely cover the original airspace.

Table.2. the comparison of the airspace before and after improvement

\begin{tabular}{|l|l|l|}
\hline \multicolumn{1}{|c|}{ model } & $M(\mathrm{~m})$ & $L(\mathrm{~m})$ \\
\hline $\begin{array}{l}\text { The airspace under the } \\
\text { traditional method }\end{array}$ & 10108 & 38713 \\
\hline $\begin{array}{l}\text { The airspace after the } \\
\text { improvement based on } \\
\text { the wind spiral method }\end{array}$ & 10757 & 39019 \\
\hline
\end{tabular}

(2) Table 2 shows us that the difference between the width $\mathrm{M}$ before or after the improvement is 649 meters, the difference between the vertical distances is $306 \mathrm{~m}$, the enlarged training airspace is more closer to the actual airspace, which can improve the safety of the flight training and reduce the conflict rate of the flying. 


\section{Conclusion}

With the $3^{\text {rd }}$ generation gradually replaces the second generation of the aircraft and becomes the major power of the air force, the need of the training becomes more frequent. In order to take full advantage of the airspace, we need to reduce the potential danger of the disturbance of other factors like wind to the delimitation of the airspace. This paper uses the wind spiral method to stimulate the turning track of the aircraft in the air combat. And also, through the comparison with the traditional track, we can know that the improved flight track is more accordant with the need of the combat flying; and that according to this, we can delimit various tactical actions flying track influenced by the wind, decide the minimum airspace needed, making the flying plan and organize the tactical drills.

\section{Acknowledgement}

In this paper, the research was sponsored by the Military Youth project of National social science foundation (Project No. 16GJ004-264) and the National Scientific Research Project of Air Traffic Control(Project No. KGKT05140501)

\section{References}

[1] Chong Yang. Simulation method for two-dimensional plane moving target track[J].Journal of Shaanxi University of Science \& Technology,2014,32(3):153-157.

[2] Li Yang, Hong An. Research on combat airplane maneuvering flight path simulation[J]. Microcomputer Information,2011,27(11):32-134

[3] Xiaohong Wang. One Universal Target Data Simulator[J].Modern Radar,2004,26(4):32-34.

[4] Rongmao He, Peng Teng, Lei Yu,etc.Flight path simulation based on movement equations under assumption of earth's surface as reference[J].Ship Electronic Engineering, 2009,29(1).122-124.

[5] Jun Huang, Peng Teng, Lei Yu,etc. Algorithm of overloading verification for same fighter's trajectory[J]. Flight Dynamics, 2008,26(3):22-25.

[6] Peng Teng, Jun Huang, Bin Zhang.Study on the three-dimensional trajectory optimization by using the method of B-spline cure fitting[J].Fire Control and Command Control,2007, 32(9):115-118.

[7] Chunmei Sun, Chaoyang Feng, Lijun Wang.Automatic creating technique of universal flight track[J]. Ordnance Industry Automation, 2010,29(12):20-25. 